\title{
Growth of Uveal Melanoma following Intravitreal Bevacizumab
}

\author{
Jasmine H. Francis ${ }^{a, b}$ Jonathan Kim ${ }^{c}$ Amy Lin ${ }^{d}$ Robert Folberg ${ }^{e}$ \\ Saipriya lyer ${ }^{a}$ David H. Abramson ${ }^{a, b}$ \\ a Ophthalmic Oncology Service, Memorial Sloan-Kettering Cancer Center, and ${ }^{\mathrm{b}}$ Department of Ophthalmology, \\ Weill Cornell Medical College, New York, NY, 'Department of Ophthalmology, University of Southern California, \\ Los Angeles, CA, d University of Illinois Hospital and Health Sciences System, Chicago, IL, and e Oakland University \\ William Beaumont School of Medicine, Rochester, MI, USA
}

\section{Keywords}

Bevacizumab · Uveal melanoma · Cancer · Intravitreal

\begin{abstract}
Purpose: Typically treatment of large melanomas (by Collaborative Ocular Melanoma Study criteria) is restricted to enucleation, due to size constraints for plaque brachytherapy. Because primary and metastatic uveal melanoma cells are inhibited by bevacizumab (an anti-vascular endothelial growth factor), this prospective study evaluated the impact of intravitreal bevacizumab on large uveal melanomas that were destined for enucleation. Size reduction by bevacizumab would potentially salvage these eyes by making them eligible for treatment with plaque brachytherapy. Procedures: Two patients with large uveal melanoma were each treated with one intravitreous injection of bevacizumab (1.25 mg/0.05 mL). Results: Both tumors displayed paradoxical growth 1 week following the injection, with confirmed growth 1 week later (increase from baseline of $1.1 \mathrm{~mm}$ in one eye and $3.1 \mathrm{~mm}$ in the other eye). Both eyes were enucleated and monosomy 3 and vasculogenic mimicry patterns were identified in both tumors. At 9 years follow-up, both patients were alive and metastasis free. Conclusion: These patients
\end{abstract}

demonstrate that neoadjuvant intravitreous bevacizumab does not decrease the size of large uveal melanomas and may, in fact, result in their paradoxical growth. This observation supports a cautious approach in the use of intravitreous bevacizumab for uveal melanoma, particularly in the neoadjuvant setting.

(c) 2016 S. Karger AG, Basel

\section{Introduction}

There is a size limit for treatment of uveal melanoma by plaque brachytherapy, predominantly restricted by the penetration of the isotope and the depth that a tumoricidal dose can be given. In the Collaborative Ocular Melanoma Study (COMS), tumors greater than $10 \mathrm{~mm}$ in height were constrained to treatment by enucleation (with or without preoperative external beam radiation to the orbit) [1]. In some fields of oncology, tumors are chemoreduced prior to radiation treatment, sometimes with the intention of reducing the field of radiation and associated dose-related toxicity. This is not routinely done in primary uveal melanoma, in part because there is no known effective chemotherapeutic agent for this tumor.

\section{KARGER}

(c) 2016 S. Karger AG, Basel

E-Mail karger@karger.com

www.karger.com/oop
Jasmine H. Francis, MD

Ophthalmic Oncology Service

Memorial Sloan-Kettering Cancer Center

1275 York Ave, New York, NY 10065 (USA)

E-Mail francij1@mskcc.org 
However, it is known that uveal melanoma expresses vascular endothelial growth factor (VEGF) [2]. In addition, bevacizumab (a recombinant humanized monoclonal antibody that acts as anti-VEGF agent) can suppress the growth of both uveal melanoma and hepatic metastases of uveal melanoma in a dose-dependent manner in an animal model [3]. Furthermore, intravitreal injections of bevacizumab allow for penetration of drug into the choroid [4]. Therefore, we theorized whether intravitreous bevacizumab could shrink large uveal melanomas in eyes restricted to enucleation, with the potential of reducing the tumor to a size amenable to treatment with plaque brachytherapy. We herein report on a prospective trial using intravitreous bevacizumab as neoadjuvant treatment in eyes with large uveal melanomas destined for enucleation.

\section{Methods}

This prospective study was approved by the Institutional Review Board of Memorial Sloan Kettering (\#07-040, NCT00596362) and patients signed informed consent prior to inclusion in the study. The study included patients aged 18 years or older with large uveal melanoma defined as tumor thickness $>10 \mathrm{~mm}$ or basal diameter $>16 \mathrm{~mm}$ (as measured by ultrasound, fundoscopic exam, or transillumination), who elected to undergo an enucleation. Two patients were enrolled in the study and no additional patients were enrolled due to paradoxical growth of tumor in the two initially enrolled patients, which led to closing the study prematurely. Prior to enucleation and during the preoperative period (systemic workup and presurgical clearance), patients received an intravitreous injection of bevacizumab $(1.25 \mathrm{mg} / 0.05 \mathrm{~mL})$ to a tumor-free quadrant of the eye by standard injection procedure. The tumor was evaluated weekly using indirect ophthalmoscopy, ultrasound, transillumination, and slit-lamp examination (with gonioscopy if indicated). The tumor height was evaluated on two sequential, weekly visits: if the tumor height remained the same or increased, the eye would be enucleated, and if the tumor decreased in height to that of a COMS medium-sized melanoma, the option of plaque brachytherapy would be offered to the patient. Enucleated specimens were histopathologically evaluated and cytogenetic analysis by fluorescence in situ hybridization was performed by a previously reported method [5]. On one specimen, angiogenic hot spots were evaluated by immunohistochemistry to endothelial cell markers CD34, CD31, factor 8, and CD105.

\section{Results}

\section{Case 1}

A 52-year-old woman was diagnosed with a choroidal melanoma in the right eye measuring $14 \mathrm{~mm}$ in diameter and $11.4 \mathrm{~mm}$ in height by ultrasonography. Magnetic res- onance imaging of the abdomen revealed no evidence of metastatic disease and the patient elected to enroll in the present study. She received one intravitreous bevacizum$\mathrm{ab}$ injection to the right eye. At 1 week following the injection, the tumor height had increased to $11.8 \mathrm{~mm}$, and 1 week later, the tumor had increased to $12.5 \mathrm{~mm}$. The eye was subsequently enucleated 2 months later, at which point the height measured $15.0 \mathrm{~mm}$ (Fig. 1). On pathology, the tumor was composed of spindle cells, had $5 \mathrm{mi}-$ totic figures per 40 high-power fields, fewer than 100 tumor-infiltrating lymphocytes in 40 high-power fields, and contained vasculogenic mimicry patterns (Fig. 2). Monosomy 3 was identified in $70 \%$ of cells. At 9 years follow-up, the patient remains alive and metastasis free.

\section{Case 2}

A 35-year-old man was diagnosed with a ciliochoroidal melanoma in the right eye measuring $10 \mathrm{~mm}$ in diameter and $11.5 \mathrm{~mm}$ in height by ultrasonography. Magnetic resonance imaging of the abdomen revealed no evidence of metastatic disease and the patient elected to enroll in the present study. He received one intravitreous bevacizumab injection to the right eye. At 1 week following the injection, the tumor height had increased to 14.5 $\mathrm{mm}$, and 1 week later, the tumor was found to measure $14.6 \mathrm{~mm}$ and the eye was enucleated (Fig. 1). On pathology, the tumor was composed of mixed (predominantly epithelioid) cells, had 4 mitotic figures per 40 high-power fields, fewer than 100 tumor-infiltrating lymphocytes in 40 high-power fields, and contained vasculogenic mimicry patterns (Fig. 2). Immunostaining with CD31 demonstrated a possible focal angiogenic hot spot adjacent to an incorporated choroidal blood vessel; CD34, CD105, and factor 8 did not show evidence of angiogenic hot spots. Monosomy 3 was identified in $76 \%$ of cells. At 9 years follow-up, the patient remains alive and metastasis free.

\section{Discussion}

Based on equivocal survival rates demonstrated in the COMS, medium-sized uveal melanomas can be treated with enucleation or plaque brachytherapy [6]. However, due to tumor size restrictions for radioactive iodine seeds, large uveal melanomas are conventionally treated by enucleation (although they can be controlled with plaque radiation in some cases [7]). In this study, two eyes with large uveal melanomas were treated with a single injection of bevacizumab prior to enucleation with the hy- 
Fig. 1. Clinical images. Case 1: color fundus photography of amelanotic uveal melanoma at presentation (upper left) and subsequent imaging 2 months later demonstrating tumor growth (upper right). Case 2: Bscan ultrasonography of intraocular melanoma at presentation (lower left) and subsequent ultrasonography 1 week following intravitreal bevacizumab demonstrating growth (lower right).

Fig. 2. Histopathology. Case 1: uveal melanoma with inconspicuous, scattered small vessels within the tumor (hematoxylin-eosin stain, reference bar $=250 \mu \mathrm{m}$ ) (upper left) highlighted by CD31 immunostain (CD31 stain, reference bar $=250 \mu \mathrm{m})$ (upper right). Case 2: uveal melanoma with dilated, ectatic vessels and focal necrosis (left lower corner) within the tumor (hematoxylin-eosin stain, reference bar $=1 \mathrm{~mm}$ ) (lower left). CD31 immunostain highlights dilated vessels seen on hematoxylin-eosin, as well as some straight vessels with compressed lumen (arrow) (CD31 stain, reference bar $=1 \mathrm{~mm})$ (lower right).
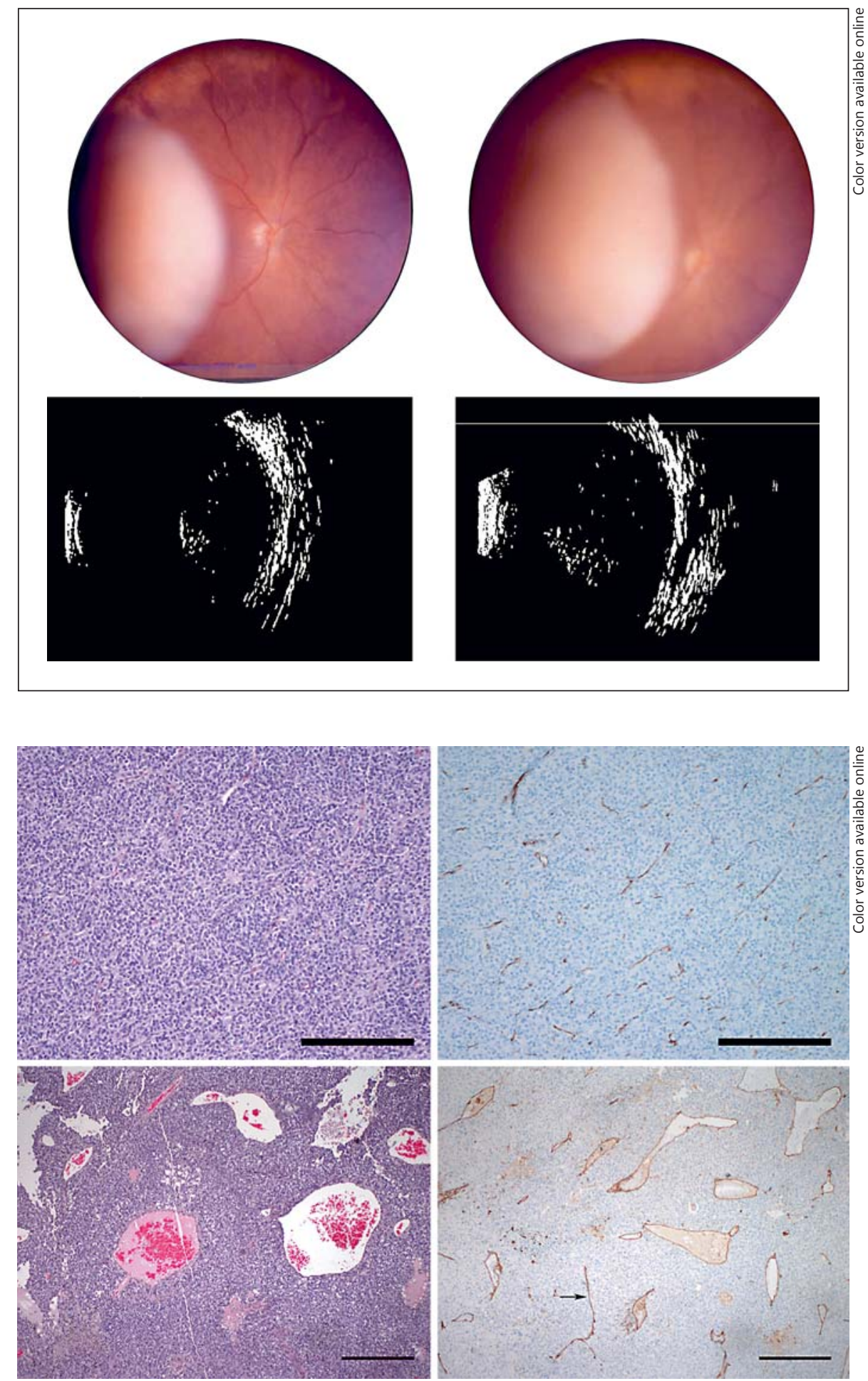

pothesis of inducing tumor reduction. However, in both patients, tumors demonstrated growth following the injections: at the time of enucleation, one tumor had increased in size by $31 \%$ while the tumor in the second eye increased by $26 \%$. This is consistent with a prior study that demonstrated continuous progression of uveal melanoma in eyes treated with multiple anti-VEGF injections for presumed choroidal neovascular membrane [8]. 
There is evidence to suggest an upregulation of VEGF in uveal melanoma and in accordance to this, suppression of both primary and metastatic uveal melanoma cells by anti-VEGF bevacizumab $[3,9,10]$. However, there is also evidence demonstrating the opposite phenomenon. In one study, in vitro exposure of B16F10 and human uveal melanoma cells led to paradoxical VEGF-A upregulation, and intraocular bevacizumab stimulated B16F10 melanoma growth in murine eyes [11].

Paradoxical effects of VEGF are thought to occur through splice variants of human VEGF-A, which inhibit (not promote) angiogenesis. For instance, VEGFA165b is a splice variant which acts in the negative regulation of angiogenesis, and in the presence of VEGFblockage, can result in vessel growth [12]. Although bevacizumab has been used for the treatment of metastases of several malignancies, levels of the splice variant VEGF-A165b can have prognostic value. For instance, low serum levels of this isoform were predictive of prolonged progression-free survival in metastatic colorectal cancer patients treated with bevacizumab [13]. Not all VEGF isoforms act to promote angiogenesis and some isoforms inhibit cell migration and proliferation in vitro [14], which may explain some of the paradoxical effects of VEGF or anti-VEGF therapy that have been reported in the literature.

It is intriguing to find that both tumors in this series were monosomy 3 and exhibited vasculogenic mimicry patterns: genetic and histopathological findings that have been associated with an aggressive clinical course $[15,16]$. However, it should be emphasized that both patients in this study are alive and metastasis free 9 years following enucleation. It begs the question as to whether these tumors, with a relatively unaggressive clinical course, would have exhibited a vasculogenic mimicry pattern had they not been exposed to intravitreous bevacizumab. Recent evidence does suggest that melanoma cells receiving
VEGF-A inhibition undergo an adaptive resistance and adopt vasculogenic mimicry patterns as an alternative tumor perfusion strategy [17].

The question remains as to whether an alternate mode of delivery of anti-VEGF (such as intra-arterially) would optimize distribution of the drug and yield different results. It is also possible that the continued growth observed was due to the natural history of uveal melanoma. However, there is evidence to suggest the need for caution when using anti-VEGF agents for uveal melanomas. In summarizing their study that demonstrated paradoxical growth of melanoma cells, el Filali et al. [11] write that the use of intraocular bevacizumab for treatment of radiation retinopathy in eyes with uveal melanoma "should be considered carefully, because of the possible adverse effects on residual UM cells." Based on the results of this present study, where uveal melanoma in two patients neither decreased nor stabilized in size but instead demonstrated growth, the same caution may be warranted in the neoadjuvant use of intravitreous anti-VEGF therapies.

\section{Acknowledgements}

This work was supported by the Fund for Ophthalmic Knowledge and Cancer Center Support Grant (P30 CA008748), who contributed to the design and conduct of the study.

\section{Statement of Ethics}

This prospective study was approved by the Institutional Review Board of Memorial Sloan Kettering (\#07-040, NCT00596362) and patients signed informed consent prior to inclusion in the study.

\section{Disclosure Statement}

None of the authors have any financial disclosures or conflicts.

\section{References}

1 Hawkins BS; Collaborative Ocular Melanoma Study Group: The Collaborative Ocular Melanoma Study (COMS) randomized trial of preenucleation radiation of large choroidal melanoma: IV. Ten-year mortality findings and prognostic factors. COMS report number 24 . Am J Ophthalmol 2004;138:936-951.

2 Sheidow TG: Expression of vascular endothelial growth factor in uveal melanoma and its correlation with metastasis. $\mathrm{Br} \mathrm{J} \mathrm{Ophthalmol}$ 2000;84:750-756.
3 Yang H, Jager MJ, Grossniklaus HE: Bevacizumab suppression of establishment of micrometastases in experimental ocular melanoma. Invest Ophthalmol Vis Sci 2010;51: 2835-2842.

4 Heiduschka P, Fietz H, Hofmeister S, Schultheiss S, Mack AF, Peters S, et al: Penetration of bevacizumab through the retina after intravitreal injection in the monkey. Invest Ophthalmol Vis Sci 2007;48:2814-2823.
5 Fang Y, Wang X, Dusza S, Jhanwar S, Abramson D, Busam KJ: Use of fluorescence in situ hybridization to distinguish metastatic uveal from cutaneous melanoma. Int J Surg Pathol 2012;20:246-251.

6 Collaborative Ocular Melanoma Study Group: The COMS randomized trial of iodine 125 brachytherapy for choroidal melanoma: $\mathrm{V}$. Twelve-year mortality rates and prognostic factors: COMS report No. 28. Arch Ophthalmol 2006;124:1684-1693. 
7 Puusaari I, Heikkonen J, Summanen P, Tarkkanen A, Kivelä T: Iodine brachytherapy as an alternative to enucleation for large uveal melanomas. Ophthalmology 2003;110:22232234.

8 Lima BR, Schoenfield LR, Singh AD: The impact of intravitreal bevacizumab therapy on choroidal melanoma. Am J Ophthalmol 2011; 151:323-328.e2.

9 Boyd SR, Tan D, Bunce C, Gittos A, Neale $\mathrm{MH}$, Hungerford JL, et al: Vascular endothelial growth factor is elevated in ocular fluids of eyes harbouring uveal melanoma: identification of a potential therapeutic window. Br J Ophthalmol 2002;86:448-452.

10 Yang M, Kuang X, Pan Y, Tan M, Lu B, Lu J, et al: Clinicopathological characteristics of vascular endothelial growth factor expression in uveal melanoma: a meta-analysis. Mol Clin Oncol 2014;2:363-368.
11 el Filali M, Ly LV, Luyten GPM, Versluis M, Grossniklaus HE, van der Velden PA, et al: Bevacizumab and intraocular tumors: an intriguing paradox. Mol Vis 2012;18:24542467.

12 Kawamura H, Li X, Harper SJ, Bates DO, Claesson-Welsh L: Vascular endothelial growth factor (VEGF)-A165b is a weak in vitro agonist for VEGF receptor-2 due to lack of coreceptor binding and deficient regulation of kinase activity. Cancer Res 2008;68:46834692.

13 Bates DO, Catalano PJ, Symonds KE, Varey AHR, Ramani P, O’Dwyer PJ, et al: Association between VEGF splice isoforms and progression-free survival in metastatic colorectal cancer patients treated with bevacizumab. Clin Cancer Res 2012;18:6384-6391.
14 Rennel E, Waine E, Guan H, Schüler Y, Leenders W, Woolard J, et al: The endogenous anti-angiogenic VEGF isoform, VEGF165b inhibits human tumour growth in mice. Br J Cancer 2008;98:1250-1257.

15 Yang JP, Liao YD, Mai DM, Xie P, Qiang YY, Zheng LS, et al: Tumor vasculogenic mimicry predicts poor prognosis in cancer patients: a meta-analysis. Angiogenesis 2016;19:191200.

16 Maniotis AJ, Folberg R, Hess A, Seftor EA, Gardner LM, Pe'er J, et al: Vascular channel formation by human melanoma cells in vivo and in vitro: vasculogenic mimicry. Am J Pathol 1999;155:739-752.

17 Schnegg CI, Yang MH, Ghosh SK, Hsu M-Y: Induction of vasculogenic mimicry overrides VEGF-A silencing and enriches stem-like cancer cells in melanoma. Cancer Res 2015; $75: 1682-1690$ 